\title{
Patient taciturnity in health counselling was understood in terms of 4 participation frames
}

\author{
Kettunen T, Poskiparta M, Liimatainen L, et al. Taciturn patients in health counseling at a hospital: passive recipients or \\ active participators? Qual Health Res 2001 May;11:399-422.
}

\section{QUESTION: How do taciturn (silent) patients communicate during hospital counselling sessions?}

\author{
Design \\ Qualitative study using an adaptation of conversation \\ analysis to analyse data. \\ Setting \\ A hospital in Finland.

\section{Patients} \\ 38 patients (age range $18-70$ y, 63\% women) and 19 \\ nurses who participated in 38 nurse-patient health \\ counselling sessions in 7 different wards.
}

\section{Methods}

Genuine patient counselling sessions related to admission, discharge, preoperative issues, and patient illness were followed up with individual interviews. Videotapes were transcribed verbatim, including stammering, and supplemented with information about periods of silence, overlapping speech, intonation, and some non-verbal communication. The tapes and transcripts were analysed using conversation analysis to examine how "turns" were taken with regard to the other participant's speech and the implications each turn had for the next.
Sources of funding: Ministry of Health and Social Affairs of Finland and the Finnish Cultural Foundation.

For correspondence: Ms T Kettunen,

Department of Sport and Health Sciences,

University of Jyväskylä,

Finland.

\section{Main findings}

The findings focused on 18 patients who were identified as taciturn or silent. They spoke little about themselves, did not introduce new topics, and supported the manner and theme of discussion chosen by nurses. 4 participation frames that produced taciturnity were identified. Participation frame is a metaphor that refers to setting and attempts to convey that the interaction is influenced by unstated rules or principles.

\section{COMMENTARY}

The qualitative study by Kettunen et al provides a rare look at the contribution of silent patients to the interaction between patients and nurses. It provides an interpretation of communication by patients who are often viewed as passive, helpless, timid, or regressive in nature. Although nurses used some helpful techniques, they also used communication techniques that did not facilitate communication and were harmful to a therapeutic relationship. These findings call into question the use of structured interview forms and teaching using a standardised format without first evaluating patients' knowledge and incorporating more helpful communication techniques.

Several strategies increased the rigour of the study. Triangulation of methods included the use of videotapes, verbatim transcripts, and interviews with participants. Member checking was used to ensure credibility, and expert peer review was used to validate findings. Finnish culture may have influenced patient responses. Transferability might be limited to similar institutions and patients from the same culture.

The study has strong implications for nursing practice. Nurse-patient communication needs to be patient driven, not guided by tradition, and based on the knowledge and experience of each patient's needs and wishes. Interactions should empower patients and not make them dependent on healthcare professionals.

Sarah Mynatt, RN, CS, EdD Professor and Psychiatric Family Nurse Option Coordinator College of Nursing, The University of Tennessee Health Science Center Memphis, Tennessee, USA,
The first frame, in the hands of professionals, was constructed from manifestations of trust in patients' speech. It was seen in small scale checking of instructions or information and comments about routine care and being the objects of care. Patient speech implied familiarity with hospital activities and questions usually focused on small details. This frame represents patients' rational view that they expect to be treated by qualified, competent caregivers.

The second frame, compliant, was constructed of responsive participants as well as recipients of information and attentive listeners. This frame emphasises the nurses' role because "patients have no opportunity to break down the nurses' benevolent authority". It was observed in nurses imparting general information or emphasising their position as formal experts through the use of institutional jargon or standardised discussion formats. The interaction was determined by the professional role of nurses, through which they tried to influence patients' health behaviour. Nurses' questions were often restrictive and focused on forms; patients seldom interrupted and responses were brief and confirmatory.

The third participation frame, guilty, was seen when patients presented themselves as incompetent to care for their own health and when nurses persuaded and questioned patients. An example occurred during exercise counselling with a patient who had had cardiac bypass surgery. The patient revealed her guilt about not exercising enough before her surgery. In response, the nurse consoled her by emphasising that exercise is only one of many contributing factors, but then confirmed the patient's guilt by arguing about the importance of being fit. The nurse then moved into a line of questioning similar to what teachers do in a classroom when they already know an answer - teacher asks, student answers, teacher approves the answer.

In the fourth participation frame, polite, patients were constructed as quiet survivors with practical experience and knowledge, who expressed their opinions and wishes. They were happy to report what was going well, which may help to build a positive outlook or to protect themselves. This frame often included humorous comments by patients to "save face." It was clear that patients experienced their problems, but they did not necessarily recount their experiences - rather they simply focused on the end result. Patients often brought up important issues through indirect hinting, but seldom interrupted nurses.

\section{Conclusion}

Patient taciturnity in health counselling situations was complex, supported by the hospital's institutional standards, nurses' lack of expertise, and patients' restrictive and face saving speech. 